\title{
SFM Assisted In-Situ by ToF-SIMS: Accessing Chemical Information in True Three Dimensions
}

\author{
Raphaëlle Dianoux ${ }^{1}$, Adi Scheidemann ${ }^{1}$ \\ Ewald Niehuis $^{2}$, Rudolf Möllers ${ }^{2}$, Felix Kollmer ${ }^{2}$, Henrik Arlinghaus ${ }^{2}$ \\ Hans-Josef Hug $^{3}$, Laetitia Bernard ${ }^{3}$, Sasa Vranjkovic ${ }^{3}$ \\ ${ }^{1}$ NanoScan AG, Duebendorf, Switerzland \\ ${ }^{2}$ ION-TOF GmbH, Muenster, Germany \\ ${ }^{3}$ Empa, Duebendorf, Switzerland
}

Scanning Force Microscopy (SFM) is a well-established tool for surface analysis in research and industry around the world nowadays. Originally developed to image atoms, the range of applications has since been widely deployed to make it not only an imaging technique but also a sensor of local forces of the surface and a manipulator of atoms and molecules. Very sharp, functionalized tips mounted on micro-levers constitute extremely sensitive sensors to the local force field emanating from the surface and thus make the SFM a general tool for the analysis of physical properties at the nanoscale.

Operated in dynamic, multi-frequency mode, the SFM can discern between concurring forces. For instance, in Magnetic Force Microscopy (MFM), magnetic properties can be recorded simultaneously to topographical data, with a lateral resolution down to $10 \mathrm{~nm}$ [1]. In Kelvin probe Force Microscopy (KPFM), the contact potential difference between tip and sample is compensated while simultaneously measuring an artifact-free topography. Vacuum operation enhances sensitivity of dynamic SFM by a factor of ca. 20, as the quality factor of the cantilever is increased by a factor of 100 or more.

SFM is a non-destructive method yielding calibrated forces in the pico to the nano-Newton range, with a lateral resolution ranging from atomic scale to the nanometer and with a sub-nanometer vertical range. However, except in rare cases [2], it cannot identify unequivocally the chemical origin of the surface, nor can it give insight into the depth of the sample. Time-of-Flight Secondary Ion Mass Spectrometry (ToF-SIMS) is the method of choice to remedy these limitations. ToF-SIMS provides chemical information at the elemental as well as the molecular level with a sensitivity up to the ppb range and a lateral resolution down to $20 \mathrm{~nm}$. For depth profiling applications, a second, dedicated sputtering beam is used to carry out sample erosion. In this so-called dual beam mode, a depth resolution below $1 \mathrm{~nm}$ can be achieved [3].

We have combined the techniques of SFM and ToF-SIMS in one UHV analysis chamber. This instrument was conjointly developed within the framework of a FP7 research project [4]. One of the key components of the new instrument is a piezo driven XYZRT-stage which moves the sample between the TOF-SIMS and the SFM analysis site with sub- $\mu \mathrm{m}$ precision and high speed. This allows analyzing exactly the same surface area with both techniques.

The SFM unit is cantilever-based and equipped with a 4-quadrant Optical Beam Deflection System (OBDS). The flexure stage is a fully-linearized scanner with $80 \times 80 \times 10 \mu \mathrm{m}^{3}$ range driven by a 20-bit digital control. Scanner and piezo stage movements are correlated to allow for the measurement of large-area images surpassing the scan range, or of long profiles across sputter craters. Crater depth is of crucial importance to localize vertically successive ToF-SIMS images. The SFM controller is further 
equipped with two Phase-Lock-Loops (PLL) which allow for dual-frequency demodulation in dynamic mode. It also features a KPFM module.

The TOF-SIMS analysis is performed using a new bismuth liquid metal cluster ion gun that can achieve a beam size for Bi3 cluster primary ions down to $20 \mathrm{~nm}$ [5]. These heavy projectiles exhibit very high secondary ion yields in particular for organic materials. For sputtering, the instrument is equipped with both an oxygen and a cesium beams. At low sputter energies of a few hundred eV a depth resolution of about $1 \mathrm{~nm}$ is achieved for inorganic thin films.

In this work, we aim at showing how ToF-SIMS is complementary to SFM on the two aspects of chemical information and of 3-dimensional analysis by presenting results on reference samples.

First, an application of KPFM is shown on a patterned sample consisting of layers of silicon, aluminum and copper (see Figure 1). Contact potential differences (CPD) are clearly identified for the 3 layers (Fig. 1b), however they cannot be assigned to a specific element. Only with the ToF-SIMS analysis is the identification unequivocal (Fig. 1c).

Second, the in-depth magnetism of a conventional hard disk is imaged using MFM. As layers are being sputtered away, the magnetic contrast becomes first stronger due to the removal of the lubricating layer, but then disappears as the magnetic layer is progressively removed.

Finally, an OLED unit cell is analyzed in-depth by successive ToF-SIMS and SFM topographical analyses of the sputtered slices. On such a complex structure, different sputtering rates lead to a depth differing widely between homogeneous regions. SFM is thus essential to reconstruct the correct volume of the cell.
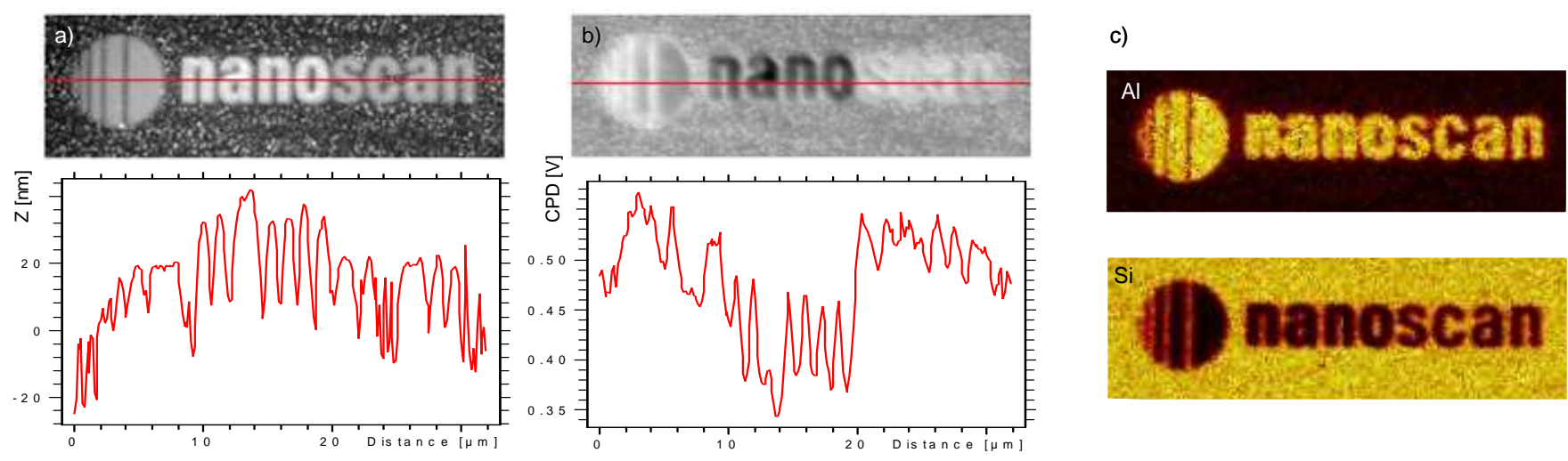

Figure 1: a) $32 \times 10 \mu \mathrm{m}^{2} \mathrm{SFM}$ topography of a patterned Al/Cu/Si surface. b) Simultaneous KPFM signal measuring the contact potential difference. c) ToF-SIMS images of aluminum and silicon.

\section{References:}

[1] B.C. Stipe et al. Nature Photonics 4 (2010), p. 484

[2] Y. Sugimoto et al. Nature 446 (2007), p. 64

[3] K. Iktgen et al. in "Secondary Ion Mass Spectrometry", SIMS X., ed. A. Bennighoven, B. Hagenhoff and H.W. Werner, (John Wiley \& Son)

[4] The authors acknowledge funding from the European Commission (FP7, Grant number 200613).

[5] F. Kollmer et al. , Surf. Interface Anal. 45 (2013), p. 312 